\title{
Benign Neoplasm of the Meninges
}

National Cancer Institute

\section{Source}

National Cancer Institute. Benign Neoplasm of the Meninges. NCI Thesaurus. Code C4957.

A benign tumor occurring in the meninges, which surround the brain and spinal cord. The most common are meningiomas. - 2004 